\title{
Comparative evaluation of the efficiency of 1 comenate the BG-Sentinel trap, CDC light trap and Mosquito-oviposition trap for the surveillance of vector mosquitoes
}

Yiji Li', Xinghua Su${ }^{1}$, Guofa Zhou ${ }^{2}$, Hong Zhang ${ }^{1}$, Santhosh Puthiyakunnon ${ }^{1}$, Shufen Shuai ${ }^{1}$, Songwu Cai ${ }^{3}$, Jinbao Gu', Xiaohong Zhou', Guiyun Yan ${ }^{1,2}$ and Xiao-Guang Chen ${ }^{1 *}$

\begin{abstract}
Background: The surveillance of vector mosquitoes is important for the control of mosquito-borne diseases. To identify a suitable surveillance tool for the adult dengue vector Aedes albopictus, the efficacy of the BG-Sentinel trap, CDC light trap and Mosquito-oviposition trap (MOT) on the capture of vector mosquitoes were comparatively evaluated in this study.

Methods: The capture efficiencies of the BG-Sentinel trap, CDC light trap and Mosquito-oviposition trap for common vector mosquitoes were tested in a laboratory setting, through the release-recapture method, and at two field sites of Guangzhou, China from June 2013 to May 2014. The captured mosquitoes were counted, species identified and compared among the three traps on the basis of species.

Results: In the release-recapture experiments in a laboratory setting, the BG-Sentinel trap caught significantly more Aedes albopictus and Culex quinquefasciatus than the CDC light trap and Mosquito-ovitrap, except for Anopheles sinensis. The BG-Sentinel trap had a higher efficacy in capturing female rather than male Ae. albopictus and $C X$. quinquefasciatus, but the capture in CDC light traps displayed no significant differences. In the field trial, BG-Sentinel traps collected more Aedes albopictus than CDC light traps and MOTs collected in both urban and suburban areas. The BG-Sentinel trap was more sensitive for monitoring the population density of Aedes albopictus than the CDC light trap and MOT during the peak months of the year 2013. However, on an average, CDC light traps captured significantly more $C X$. quinquefasciatus than BG-Sentinel traps. The population dynamics of $C$. quinquefasciatus displayed a significant seasonal variation, with the lowest numbers in the middle of the year.

Conclusions: This study indicates that the BG-Sentinel trap is more effective than the commonly used CDC light trap and MOT in sampling adult Aedes albopictus and Culex quinquefasciatus. We recommend its use in the surveillance of dengue vector mosquitoes in China.
\end{abstract}

Keywords: BG-Sentinel trap, CDC light trap, Mosq-ovitrap, Aedes albopictus, Culex quinquefasciatus, Anopheles sinensis, Surveillance

\footnotetext{
* Correspondence: xgchen2001@hotmail.com

${ }^{1}$ Key Laboratory of Prevention and Control for Emerging Infectious Diseases

of Guangdong Higher Institutes, Department of Pathogen Biology, School of

Public Health and Tropical Medicine, Southern Medical University,

Guangzhou North Avenue No.1838, Guangzhou 510515, China

Full list of author information is available at the end of the article
} 


\section{Background}

Mosquitoes are vectors of various human and animal diseases, such as malaria, filariasis, dengue fever, Chikungunya fever, Japanese encephalitis, and yellow fever [1]. Vector control is an essential component and sometimes is the only effective way (e.g. for dengue fever) to block or reduce the transmission of these diseases [2-5]. Using a sensitive and efficient surveillance tool to monitor the species composition and population dynamics of local mosquitoes is the most important step in developing and implementing appropriate strategies to control vector populations.

At present, there are already several methods or techniques to survey the population and density of vector mosquitoes [6-13]. However, varied efficacies have been reported for different types of traps [14, 15]. Currently, the commonly used methods in surveillance programmes to collect adult mosquitoes in China include the Centre for Disease Control and Prevention (CDC) light trap, Mosquito-oviposition trap (MOT) and human landing catches. CDC light traps are the most commonly used method for the surveillance of mosquito populations, and many previous reports have shown that it is effective in capturing Culex and Anopheles but not Ae. albopictus $[16,17]$. Aedes albopictus is a daytime-biting (from dawn to dusk) mosquito species and is the primary vector of dengue fever in China [18]. A previous report has indicated that the CDC light trap is not efficient for the surveillance of Ae. albopictus [19]. MOTs are primarily used to collect eggs and female adults of Ae. albopictus, but the efficacy in collecting adult mosquitoes is low [20]. Although the human landing catch is a very effective way to catch adult mosquitoes, especially Aedes mosquitoes [21, 22], it leads to serious ethical concerns associated with the use of humans as bait because of the potential risk of infection with dengue viruses. All these facts indicate the lack of a safe, standard and sensitive method for vector mosquito surveillance in China, especially for the dengue vector mosquito, Ae. albopictus.

In recent years, BG-Sentinel traps (BioGents Corporation, Regensbourg, Germany) have been used to collect Aedes (Stegomyia) mosquitoes such as Ae. aegypti, Ae. albopictus, and Ae. polinesiensis [23-26]. BGS traps can be used with a variety of mosquito attractants, e.g. $\mathrm{CO}_{2}$, BG-lure, and octanol, thereby making it a versatile tool for mosquito research and surveillance. Nevertheless, there is no report on evaluating the efficiency of the BG-Sentinel trap in comparison with other traps for the surveillance of dengue vector mosquitoes in China.

In this study, we evaluated the efficacy of the BGSentinel trap, CDC light trap and MOT traps in terms of mosquito captures by species in a laboratory setting as well as in the fields of Guangzhou, Guangdong province, China.

\section{Methods}

\section{Description of study sites}

Laboratory-based experiments were carried out at the Centre for Disease Control and Prevention of Guangdong Province, Guangzhou, China, and field trials were conducted from June 2013 to May 2014 in Tonghe and Liangtian of Guangzhou. Guangzhou is the largest city in Southern China, with a population of 12 million according to the 2012 census. Guangzhou has been the major region of dengue epidemic in China in recent years [27]. The annual average temperature is $21.6{ }^{\circ} \mathrm{C}$, and rainfall is $1,983 \mathrm{~mm}$. This climate is ideal for the development and reproduction of vector mosquitoes.

Tonghe $\left(113^{\circ} 19^{\prime} \mathrm{E}, 23^{\circ} 11^{\prime} \mathrm{N}, 31 \mathrm{~m}\right.$ above sea level, m.a.s.l.) is an urban area with a population density of $>3,000$ people $/ \mathrm{km}^{2}$. The land use includes primarily residential and commercial buildings, as well as public services facilities, such as schools and hospitals, filled with trees and grasses. Liangtian $\left(113^{\circ} 23^{\prime} \mathrm{E}, 23^{\circ} 21^{\prime} \mathrm{N}, 25 \mathrm{~m}\right.$ a.s.l.) is a suburban area with a population density of approximately 1,000 people $/ \mathrm{km}^{2}$, and land use includes a mixture of residential, manufacturing, and farmland (Fig. 1).

\section{The traps}

Three types of traps were tested: BGS traps (BioGents, Regensbourg, Germany) with BG-Lure (BioGents, GmbH, Regensbourg, Germany), CDC light traps (Lucky Star Environmental Protection Technology Co., Ltd., Wuhan, China), and MOTs (Southeast Industrial Co. Ltd, Guangdong, China).

\section{Experimental design}

In the laboratory, a release-recapture experiment [28] was conducted in a standard room $(3.2 \times 2.9 \times 2.75 \mathrm{~m})$ to determine the collection efficiency of the three types of traps. Three species of mosquitoes, Ae. albopictus, Cx. quinquefasciatus and An. sinensis, were tested and all experimental mosquitoes were $3-5$ days post-emergence at release. We conducted the study with groups of 200, 400, and $800 \mathrm{Ae}$ des albopictus to evaluate if mosquito abundances affect the efficiency of the traps. Then we conducted the study with groups of 200 each for Aedes albopictus, Culex quinquefasciatus and Anopheles sinensis to evaluate the efficacies of different traps on different mosquitoes. It was reported that the mark-release-recapture rate is low in the field [29]. In all groups, we used a female to male ratio of 1:1. The mosquitoes were released in the room and three traps with one of each type were kept in the room for $24 \mathrm{~h}$. After $24 \mathrm{~h}$, mosquitoes collected in each trap were counted. The experiments were repeated eight times. The three traps were put diagonally in the room, to reduce design bias, the positions of traps were rotated in the room following a Latin square design after each replication. The laboratory environmental conditions were set 


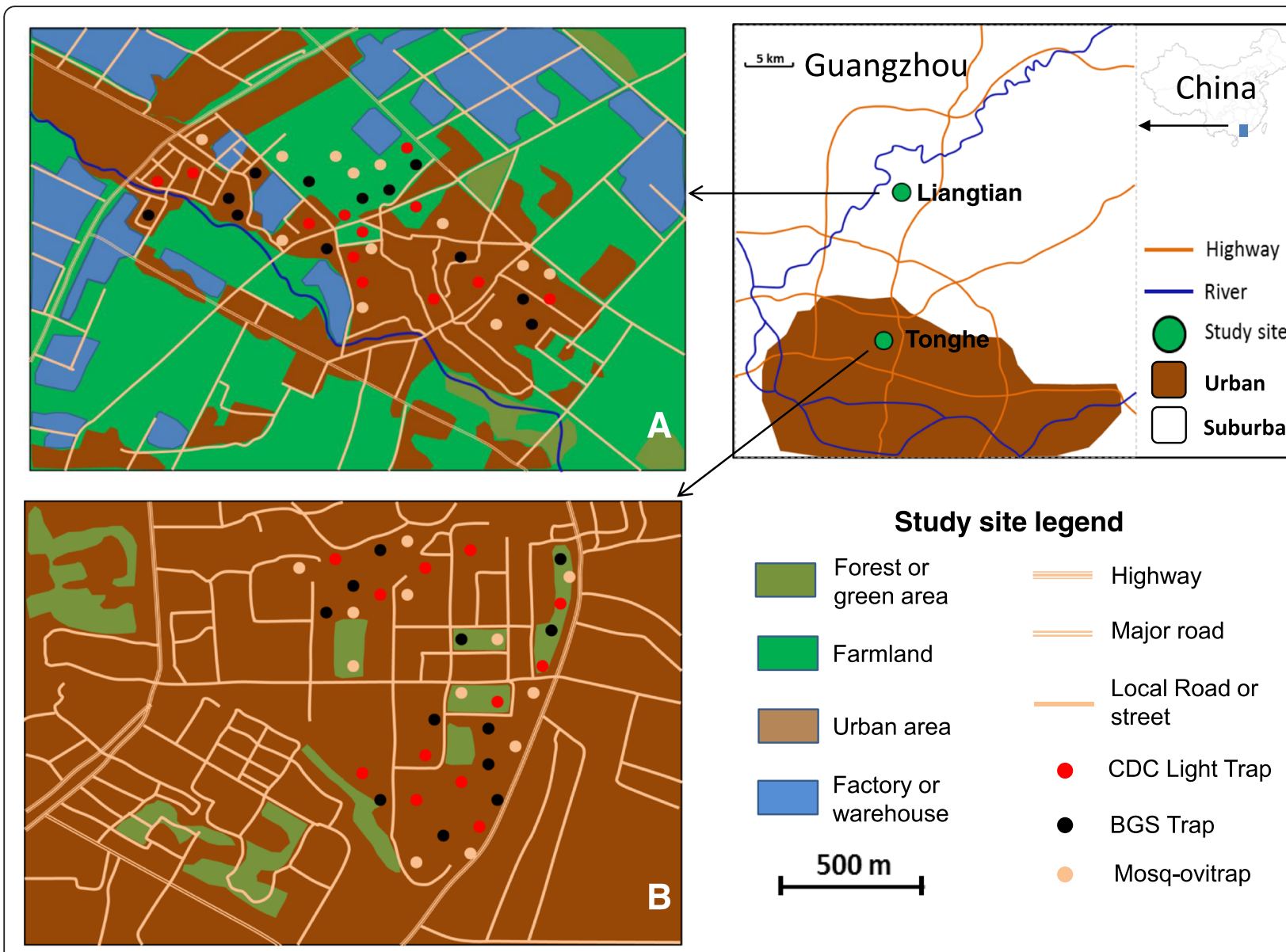

Fig. 1 Map of the study areas and distribution of the traps in the first week of each month. a Liangtian (suburban area), b Tonghe (urban area). Twelve each of BGS Traps, CDC Light Traps and MOTs were used to survey the mosquito density in Tonghe and Liangtian

at a temperature of $26 \pm 1{ }^{\circ} \mathrm{C}$, with a relative humidity of $60-80 \%$ and a light and dark period of $12 \mathrm{~h}$.

In the field trial, 12 of each of three types of traps were placed in the two study areas. In the urban area, we chose three locations for setting traps: a residential area, a public park, and a commercial district; in the suburban area, the locations were a residential area, a factory, and a garden. The distance between two traps was at least $30 \mathrm{~m}$. Traps were placed in the same location for three consecutive days during the first week of each month; and they were shifted to different locations for another three days during the third week of each month. The adult mosquito populations were monitored continuously from June 2013 to May 2014. The CDC light traps were hung in trees $0.8 \mathrm{~m}$ above the ground, whereas the BGS traps and MOTs were placed on the ground. Every $24 \mathrm{~h}$, as one trapping period, mosquitoes were collected and transported to the laboratory for species identification. The geographical coordinates of each sampling point were recorded using portable global positioning system (GPS) devices (Garmin eTrex H) (Fig. 1 and Additional file 1: Figure S1).

\section{Mosquito identification}

Frozen mosquitoes were placed on a piece of white filter paper in a Petri plate on a chill table, and the species were identified morphologically under a stereo microscope using taxonomic keys [30].

\section{Statistical analysis}

Differences in sex-specific captures among different traps and mosquito species under laboratory conditions were tested using generalized estimating equation (GEE) based Negative Binomial regression and Tukey's post-hoc honestly significant difference (HSD) tests. Differences in population dynamics between the BGS trap and CDC light trap were compared using the GEE Negative Binomial regression. Data were square-root transformed before Tukey's HSD test. Statistical analysis was performed using the JMP statistical software (JMP 9.0, SAS Institute Inc., USA) and R 3.0.1. Differences in sex ratio in field-captured mosquitoes between the BGS traps and CDC light traps were compared using the $\chi^{2}$-test or the Fisher exact test if any number was $<5$. 


\section{Results}

\section{Laboratory study}

For Ae. albopictus, regardless of the number of individuals released, BGS traps increased capture rates almost 2-fold compared to CDC light traps. CDC light traps captured approximately $20 \%$ more individuals than the MOTs $(P<0.001)$ (Fig. 2, Additional file 2: Table S1). Overall, regardless of population densities, the most efficient method of Aedes albopictus collection was the BGS trap, followed by CDC light traps and MOT. For Cx. quinquefasciatus, BGS traps caught twice the number of mosquitoes than $\mathrm{CDC}$ light traps, $\mathrm{CDC}$ light traps caught five times more mosquitoes than MOTs $(P<0.001)$ (Fig. 2, Additional file 2: Table S1). There was an exception for Anopheles sinensis. The CDC light traps captured almost three times more An. sinensis than the BGS traps and five times more than the MOTs $(P<0.001)$ (Fig. 2, Additional file 2: Table S1).

Table 1 illustrates the sex-specific capture by trap type and species. The BGS trap had a higher efficacy in capturing female rather than male Ae. albopictus and $C x$. quinquefasciatus (Tukey's HSD test, both $P<0.001$ ), but there was no difference in the captures of male and female An. sinensis (Table 1). The CDC light trap showed no difference in the captures of male and female Ae. albopictus and $C x$. quinquefasciatus, but for An. sinensis it captured more males than females (Tukey's HSD test, $P<0.001)$. The MOT showed a higher efficiency in capturing male rather than female Ae. albopictus, with 5-fold more males captured than females (Tukey's HSD test, $P<0.001)$, and there was no difference in the captures of male and female of both $C x$. quinquefasciatus and An. sinensis (Tukey's HSD test, $P>0.05$ ) (Table 1 ).

\section{Field study}

In our field study, a total of 864, 864 and 288 trapperiods with the BGS traps, CDC light traps and MOTs, respectively, were conducted from June 2013 to May 2014 in Tonghe. A total of 876, 864 and 288 trap-periods with the BGS traps, CDC light traps and MOTs, respectively, were performed from June 2013 to May 2014 in Liangtian. In Tonghe, a total of 27,174 mosquitoes were collected and identified by species. Among them, 7,600 were Ae. albopictus, 19,436 Cx. quinquefasciatus, 129 Armigeres subalbatus, and 9 Toxorhynchites splendens (Table 2). In Liangtian, a total of 52,649 mosquitoes were collected and identified by species: Ae. albopictus $(2,636), C x$. quinquefasciatus (49,730), Armigeres subalbatus (336) and Toxorhynchites splendens (17) (Table 2). During the survey period, the MOT collected only 49 adults and 573 eggs of Ae. albopictus and 6 adults and 88 eggs of $C x$. quinquefasciatus in Tonghe, with only 16 adults and 204 eggs of Ae. albopictus and 15 adults and 114 eggs of $C x$. quinquefasciatus in Liangtian. Due to the small number of captures, the MOT data were not subjected to further analysis.

Over $99 \%$ of the BGS trap and CDC light trap catches were either Ae. albopictus or Cx. quinquefasciatus. BGS traps captured 5-fold more Ae. albopictus than CDC light traps in both urban and suburban areas, whereas CDC light traps captured $25 \%$ less Cx. quinquefasciatus

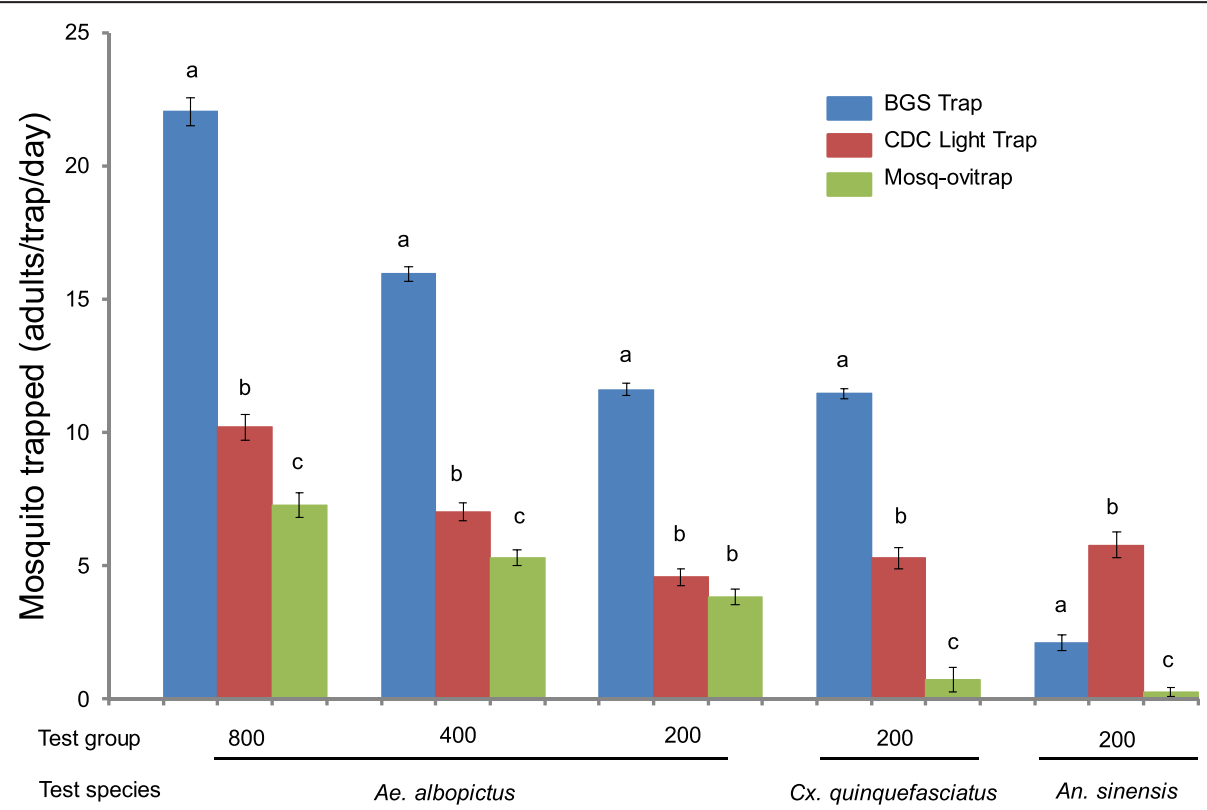

Fig. 2 The effectiveness of the three kinds of traps in catching different species of mosquitoes in laboratory conditions. Comparative analysis of mean and standard error of Aedes albopictus, CX. quinquefasciatus and An. sinensis caught in different traps under laboratory conditions. Columns with different letters are significantly different from each other $(P<0.05)$. Mosquito abundance is square-root transformed, and values are the mean \pm standard error 
Table 1 Sex differences among mosquitoes collected in the three mosquito traps in the laboratory

\begin{tabular}{|c|c|c|c|c|c|c|c|}
\hline \multirow[t]{2}{*}{ Traps } & \multirow{2}{*}{$\begin{array}{l}\text { No. of } \\
\text { release times }\end{array}$} & \multicolumn{2}{|l|}{ Ae. albopictus } & \multicolumn{2}{|c|}{ Cx. quinquefasciatus } & \multicolumn{2}{|l|}{ An. sinensis } \\
\hline & & Male & Female & Male & Female & Male & Female \\
\hline BGS trap & 8 & $7.59 \pm 0.20^{a}$ & $8.78 \pm 0.17^{b}$ & $7.50 \pm 0.19^{\mathrm{a}}$ & $8.65 \pm 0.22^{b}$ & $1.26 \pm 0.31^{a}$ & $1.53 \pm 0.24^{\mathrm{a}}$ \\
\hline CDC Light trap & 8 & $3.30 \pm 0.27^{\mathrm{a}}$ & $3.15 \pm 0.16^{a}$ & $4.09 \pm 0.24^{\mathrm{a}}$ & $3.29 \pm 0.41^{\mathrm{a}}$ & $4.77 \pm 0.44^{a}$ & $3.23 \pm 0.25^{b}$ \\
\hline Mosq-ovitrap & 8 & $3.70 \pm 0.31^{\mathrm{a}}$ & $0.78 \pm 0.24^{b}$ & $0.48 \pm 0.36^{\mathrm{a}}$ & $0.43 \pm 0.31^{a}$ & $0.11 \pm 0.13^{\mathrm{a}}$ & $0.13 \pm 0.13^{\mathrm{a}}$ \\
\hline
\end{tabular}

Rows with different letters are significantly different from each other $(P<0.05)$. Mosquito abundance is square-root transformed and values are the mean \pm standard error

Abbreviations: $M$ male, $F$ female

than BGS traps in urban areas but 2 -fold more in suburban areas (Table 2). In general, both BGS traps and CDC light traps captured significantly more males than females, regardless of species (Table 2). BGS traps captured significantly fewer female $C x$. quinquefasciatus than CDC light traps (33 vs $41 \%, \chi^{2}=124.4, d f=1, P<0.0001$ ) (Table 2).

\section{Population dynamics of the mosquitoes collected by the traps}

The population dynamics of both Ae. albopictus and $C x$. quinquefasciatus showed clear seasonal variation regardless of study site, mosquito species, and trap types (Fig. 3). The peak months varied depending on study area and species (Fig. 3). For Ae. albopictus, its captures were significantly more in urban than that in suburban area $(Z=15.91, P<0.001)$, CDC light traps captured significantly less than BGS traps $(Z=-25.13, P<0.001)$ (Fig. 3, Table 3). The population dynamics of Ae albopictus showed significant seasonal changes, with a peak collection likely in the middle of the year (Fig. 3, Table 3). In addition, in different areas, different trap types performed differently, likely due to the strong inter-site difference in population density (Table 3 ). In contrast, $C x$. quinquefasciatus were captured year round with the highest densities in both BGS and CDC light traps observed from October to December (Fig. 3). The negative binomial regression analysis revealed that, $C x$. quinquefasciatus density was significantly lower in urban than in suburban areas $(Z=-5.24, P<0.001)$, CDC light traps captured significantly more $C x$. quinquefasciatus than BGS traps $(Z=13.85, P<0.001)$ (Fig. 3, Table 3). The population dynamics of $C x$. quinquefasciatus also showed significant seasonal variations, with the lowest catch likely in the middle of the year (Fig. 3, Table 3).

\section{Discussion}

In both laboratory experiments and field surveillance tests, this study showed that BGS traps, compared to CDC light traps and MOTs, are highly efficient in capturing Ae. albopictus and $C x$. quinquefasciatus. This work highlighted the efficiency of BGS traps and their further potential for implementation in the surveillance of vector mosquitoes in China.

The laboratory experiments indicated that BGS traps are more effective in capturing female adult mosquitoes

Table 2 Species and sex composition of mosquitoes collected in BG-Sentinel and CDC light traps in Guangzhou, China

\begin{tabular}{|c|c|c|c|c|c|c|c|c|c|c|}
\hline \multirow[t]{3}{*}{ Study area } & \multirow[t]{3}{*}{ Species } & \multicolumn{8}{|c|}{ Trap method } & \multirow{3}{*}{ 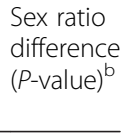 } \\
\hline & & \multicolumn{4}{|c|}{ BG-sentinel trap } & \multicolumn{4}{|c|}{ CDC light trap } & \\
\hline & & Total & Male & Female & $\mathrm{F}$ rate $(95 \% \mathrm{Cl})^{\mathrm{a}}$ & Total & Male & Female & F rate $(95 \% \mathrm{Cl})^{\mathrm{a}}$ & \\
\hline \multirow[t]{6}{*}{ Tonghe (urban) } & Number of trap days & 864 & & & & 864 & & & & \\
\hline & Aedes albopictus & 6211 & 3641 & 2570 & $0.41[0.40,0.43]$ & 1389 & 790 & 599 & $0.43[0.41,0.46]$ & 0.23 \\
\hline & Culex quinquefasciatus & 11,365 & 7588 & 3777 & $0.33[0.32,0.34]$ & 8071 & 4758 & 3313 & $0.41[0.40,0.42]$ & $<0.0001$ \\
\hline & Armigeres subalbatus & 46 & 25 & 21 & $0.46[0.32,0.60]$ & 83 & 49 & 34 & $0.41[0.31,0.52]$ & 0.60 \\
\hline & Toxorhynchites splendens & 1 & 1 & 0 & n.a. & 8 & 5 & 3 & $0.38[0.14,0.69]$ & n.a. \\
\hline & Total No. & 17,623 & 11,255 & 6368 & & 9551 & 5602 & 3951 & & \\
\hline \multirow[t]{6}{*}{ Liangtian (suburban) } & Number of trap days & 876 & & & & 864 & & & & \\
\hline & Aedes albopictus & 2181 & 1200 & 981 & $0.45[0.43,0.47]$ & 385 & 195 & 190 & $0.49[0.44,0.54]$ & 0.11 \\
\hline & Culex quinquefasciatus & 18,442 & 12,517 & 5925 & $0.32[0.31,0.33]$ & 31,288 & 16,233 & 15,055 & $0.48[0.48,0.49]$ & $<0.0001$ \\
\hline & Armigeres subalbatus & 152 & 91 & 61 & $0.40[0.33,0.48]$ & 184 & 114 & 70 & $0.38[0.31,0.45]$ & 0.70 \\
\hline & Toxorhynchites splendens & 3 & 1 & 2 & $0.67[0.21,0.94]$ & 14 & 10 & 4 & $0.29[0.12,0.55]$ & 0.51 \\
\hline & Total No. & 20,778 & 13,809 & 6969 & & 31,871 & 16,552 & 15,319 & & \\
\hline
\end{tabular}

${ }^{\mathrm{a} F}$ rate is defines as number of females over total

${ }^{b}$ Difference between the two trap-types. $X^{2}$-test or Fisher exact test if any number is $<5$ 

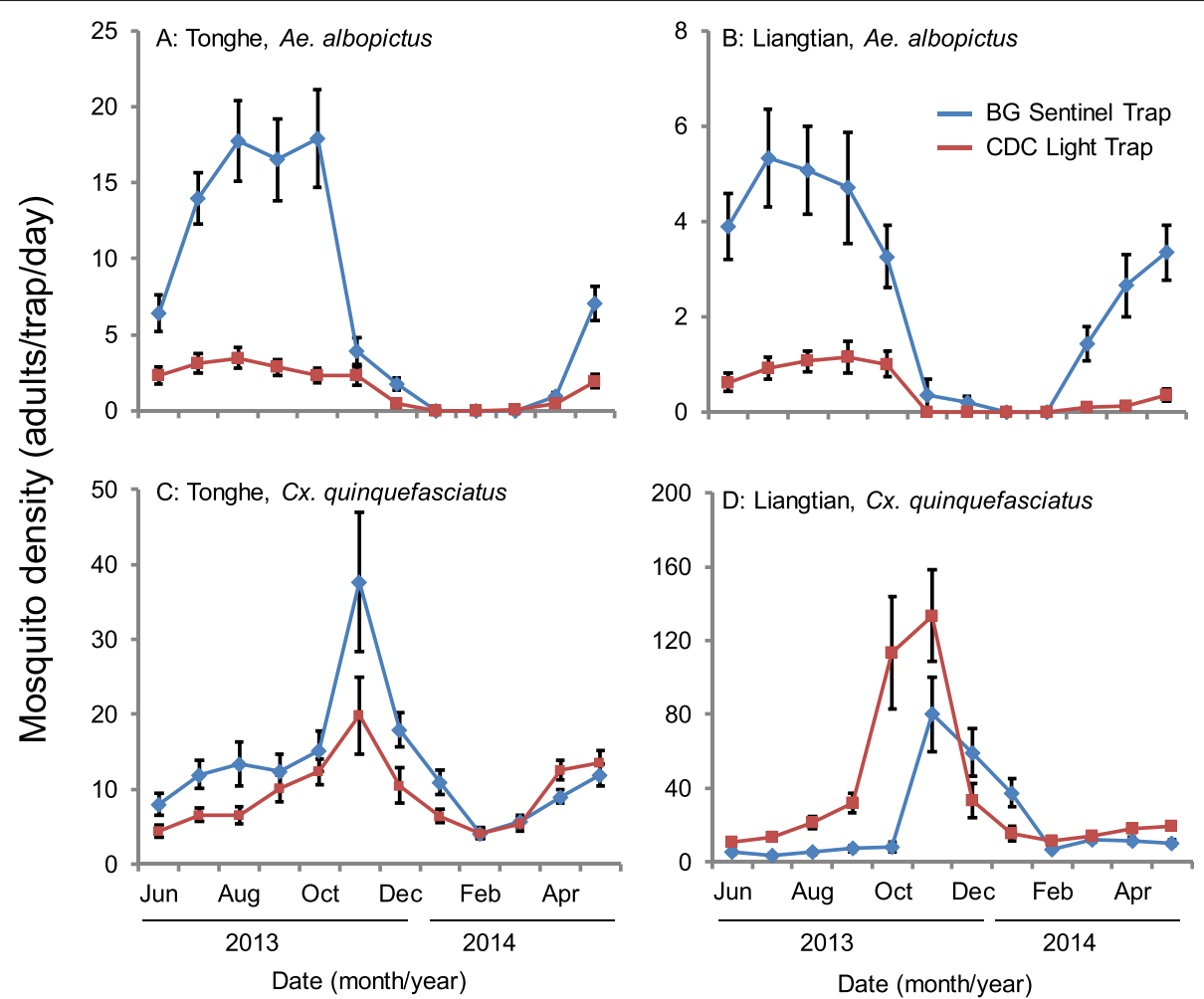

Fig. 3 Population dynamics of Aedes albopictus and Cx. quinquefasciatus collected by two traps in urban and suburban areas. a and $\mathbf{c}$ Tonghe (urban). b and d Liangtian (suburban). a and b: Ae. albopictus; c and d: CX. quinquefasciatus. Values are the mean $\pm 95 \% \mathrm{Cl}$

Table 3 Results of negative binomial regression analysis in the field study

\begin{tabular}{|c|c|c|c|c|c|}
\hline Species & Coefficients & Estimate & Std. Error & $Z$-value & $\operatorname{Pr}(>|z|)$ \\
\hline \multicolumn{6}{|c|}{ Aedes albopictus } \\
\hline & (Intercept) & -6.11 & 0.18 & -34.14 & $<0.0001$ \\
\hline & Site (urban) & 0.85 & 0.05 & 15.91 & $<0.0001$ \\
\hline & TrapType (CDC) & -1.88 & 0.07 & -25.13 & $<0.0001$ \\
\hline & $\begin{array}{l}\text { Site (urban)* } \\
\text { TrapType (CDC) }\end{array}$ & 0.51 & 0.09 & 5.41 & $<0.0001$ \\
\hline & Month & 2.07 & 0.05 & 42.20 & $<0.0001$ \\
\hline & Month*Month & -0.13 & 0.003 & -41.18 & $<0.0001$ \\
\hline \multicolumn{6}{|c|}{ Culex quinquefasciatus } \\
\hline & (Intercept) & 2.88 & 0.06 & 44.85 & $<0.0001$ \\
\hline & Site (urban) & -0.24 & 0.05 & -5.24 & $<0.0001$ \\
\hline & TrapType (CDC) & 0.62 & 0.05 & 13.85 & $<0.0001$ \\
\hline & $\begin{array}{l}\text { Site(urban) * } \\
\text { TrapType(CDC) }\end{array}$ & -0.88 & 0.06 & -13.62 & $<0.0001$ \\
\hline & Month & -0.28 & 0.02 & -13.48 & $<0.0001$ \\
\hline & Month*Month & 0.03 & 0.002 & 19.86 & $<0.0001$ \\
\hline
\end{tabular}

of Ae. albopictus and Cx. quinquefasciatus. However, the field study showed that BGS traps have a higher efficacy in capturing males rather than females of Ae. albopictus and $C x$. quinquefasciatus, which contradicts our original prediction, because BG lures consist of lactic acid, caproic acid and ammonium bicarbonate simulated human odorants that are thought to be more attractive to female mosquitoes. Similar results have also been reported from studies conducted in Brazil and Kenya [31, 32]. However, research from America showed that the ratio of male-tofemale Ae. albopictus can vary seasonally, geographically, and in response to adulticidal treatments by BGS trap with lure $[33,34]$. This phenomenon may be explained by mosquito behaviour. First, to mate with females, one shortcut for male mosquitoes is to locate potential hosts and remain nearby to increase their chances of encountering female mosquitoes [35-39]. It may be possible for male mosquitoes to recognize human odorants and use BGS traps as swarm markers. Secondly, the eclosion time for male and female mosquitoes is different. Usually, the male mosquitoes emerge first, and the females emerge afterwards. Thus, it is possible that for a period of time, the male is dominant in the environment, causing the biased attraction of BGS traps. Thirdly, male mosquitoes tend to stay in the wild, looking for nectar for food [36-39]. In this study, the traps 
were set in residential areas, public parks, commercial districts, factories, and gardens with open environmental spaces, where male mosquitoes tend to aggregate because of the abundant vegetation, whereas the females do not because of the lack of sufficient haematophagous hosts. This preference in distribution may also have caused the bias in catching.

The field surveys in this study showed that the species of mosquitoes in Guangzhou are Ae. albopictus and $C x$. quinquefasciatus, a result consistent with those from previous reports [40, 41]. Analysis of the population dynamics of the mosquitoes showed that the population density of Ae. albopictus was much higher in the urban (Tonghe) than in the suburban area (Liangtian), which is consistent with the epidemic status of dengue in Guangzhou. Our results also showed that the monitoring of Ae. albopictus by BGS trap is more sensitive than that by CDC light traps, especially during the peak season. All of these data suggested that the BGS trap is a suitable surveillance tool for the dengue vector in China.

The different traps attract mosquitoes based on various mechanisms. BG-Sentinel traps use BG-lure (human odours) to attract host-seeking mosquitoes, CDC light traps use the light to attract phototaxis mosquitoes and Mosquito-oviposition traps use the water to attract oviposition-seeking mosquitoes respectively, therefore, these traps measure very different sub-populations of the mosquitoes at different stages in their life cycle. To reduce the bias that might be caused by the different physiological states of mosquitoes, these traps were placed in the same location for three consecutive days during the first week of each month and then shifted to different locations for another three days during the third week of each month for a total of 12 months. Although in different areas, different traps performed differently, likely due to the strong inter-site difference in population density and physiological states, the final summarized results of this study displayed that BGS traps are most efficient in capturing adult Ae. albopictus and Cx. quinquefasciatus, two species of vector mosquitoes most commonly distributed in the cities of China. Considering the role of Ae. albopictus in disease transmission, we recommend using the hostseeking based BGS traps as the surveillance tool of vector mosquitoes.

\section{Conclusion}

We conducted the first comparative evaluation of the efficacy of BGS traps, CDC light traps and Mosq-ovitraps in the capture of common vector mosquitoes in a laboratory setting and in the field in Guangzhou, China. The results indicated that the BGS trap is an effective tool for the monitoring of urban vector mosquitoes and could be used in the surveillance of dengue fever in China.

\section{Additional files}

Additional file 1: Figure S1. Distribution of the traps in the third week of each month. A. Liangtian (suburban area), B. Tonghe (urban area). Twelve each of BGS Traps, CDC Light Traps and MOTs were used to survey the mosquito density in Tonghe and Liangtian. (PDF 639 kb)

Additional file 2: Table S1. Results of negative binomial regression in the laboratory study. (DOCX $15 \mathrm{~kb}$ )

\section{Acknowledgments}

We wish to thank Wenqiang Yang, Xueli Cai, Lijie Yao, Guiquan Lai and Qiankun Chen, who assisted with the field trap surveillance. We also wish to thank Jinhua Duan, Weixiong Yin and Xuguang Wu from the Centres for Disease Control and Prevention of Guangdong Province, Guangzhou for their technical support in rearing the mosquitoes for the laboratory study.

\section{Authors' contributions}

$Y L, G Y$ and $X G C$, conceived the study and coordinated its implementation. $Y L, G Z$ and $G Y$ participated in the experimental design. $Y L, X S, H Z$, SP, SS, SC, $J G$ and $X Z$ performed the experiments and drafted the manuscript which was critically revised by GZ, XGC and GY. All authors read and approved the final version of the manuscript.

\section{Competing interests}

The authors declare that they have no competing interests.

\section{Consent for publication}

Not applicable.

Ethics approval and consent to participate

Not applicable.

\section{Financial support}

This work is supported by grants from the National key research and development program (2016YFC1200504), National Institutes of Health (Al083202, D43TW009527), Natural Scientific Foundation of Guangdong Province (2013B051000052, 2014A030312016), Scientific and Technological Programme of Guangzhou (201508020263), and the Specialized Research Fund for the Doctoral Program of Higher Education (20124433110008).

\section{Author details}

${ }^{1}$ Key Laboratory of Prevention and Control for Emerging Infectious Diseases of Guangdong Higher Institutes, Department of Pathogen Biology, School of Public Health and Tropical Medicine, Southern Medical University,

Guangzhou North Avenue No.1838, Guangzhou 510515, China. ${ }^{2}$ Program in Public Health, School of Medicine, University of California, Irvine, USA.

${ }^{3}$ Department of Vector Control, Centers for Disease Control and Prevention of Guangdong Province, Guangzhou, China.

Received: 13 January 2016 Accepted: 25 July 2016

Published online: 12 August 2016

\section{References}

1. Vector Control for Malaria and Other Mosquito-Borne Disease. World Health Organization; 1995.

2. mal ERACGOHS, Operational R. A research agenda for malaria eradication: health systems and operational research. PLoS Med. 2011;8(1):e1000397.

3. van den Hurk AF, Ritchie SA, Mackenzie JS. Ecology and geographical expansion of Japanese encephalitis virus. Annu Rev Entomol. 2009;54:17-35.

4. Morrison AC, Zielinski-Gutierrez E, Scott TW, Rosenberg R. Defining challenges and proposing solutions for control of the virus vector Aedes aegypti. PLoS Med. 2008;5(3), e68.

5. Tomori O. Yellow fever in Africa: public health impact and prospects for control in the 21st century. Biomedica: revista del Instituto Nacional de Salud. 2002;22(2):178-210.

6. Bhami LC, Das SS. Boric acid ovicidal trap for the management of Aedes species. J Vector Borne Dis. 2015;52(2):147-52.

7. Caputo B, lenco A, Manica M, Petrarca V, Rosa R, della Torre A. New adhesive traps to monitor urban mosquitoes with a case study to assess the 
efficacy of insecticide control strategies in temperate areas. Parasit Vectors. 2015;8:134.

8. Casas Martinez M, Orozco Bonilla A, Munoz Reyes M, Ulloa Garcia A, Bond $J G$, Valle Mora J, et al. A new tent trap for monitoring the daily activity of Aedes aegypti and Aedes albopictus. J Vector Ecol. 2013;38(2):277-88.

9. Harwood JF, Arimoto H, Nunn P, Richardson AG, Obenauer PJ. Assessing carbon dioxide and synthetic lure-baited traps for dengue and Chikungunya vector surveillance (3). J Am Mosq Control Assoc. 2015;31(3):242-7.

10. Pombi M, Jacobs F, Verhulst NO, Caputo B, Della Torre A, Takken W. Field evaluation of a novel synthetic odour blend and of the synergistic role of carbon dioxide for sampling host-seeking Aedes albopictus adults in Rome, Italy. Parasites \& vectors. 2014;7:580.

11. Ritchie SA, Buhagiar TS, Townsend M, Hoffmann A, Van Den Hurk AF, McMahon JL, Eiras AE. Field validation of the gravid Aedes trap (GAT) for collection of Aedes aegypti (Diptera: Culicidae). J Med Entomol. 2014;51(1):210-9.

12. Wright JA, Larson RT, Richardson AG, Cote NM, Stoops CA, Clark M, Obenauer PJ. Comparison of BG-sentinel(R) trap and oviposition cups for Aedes aegypti and Aedes albopictus surveillance in Jacksonville, Florida, USA. J Am Mosq Control Assoc. 2015;31(1):26-31.

13. Xue RD, Smith ML, Yi H, Kline DL. Field evaluation of a novel mos-hole trap and naphtha compared with BG sentinel trap and mosquito magnet X Trap to collect adult mosquitoes. J Am Mosq Control Assoc. 2015;31(1):110-2.

14. Obenauer PJ, Kaufman PE, Kline DL, Allan SA. Detection of and monitoring for Aedes albopictus (Diptera: Culicidae) in suburban and sylvatic habitats in north central Florida using four sampling techniques. Environ Entomol. 2010;39(5):1608-16.

15. L'Ambert G, Ferre JB, Schaffner F, Fontenille D. Comparison of different trapping methods for surveillance of mosquito vectors of West Nile virus in Rhone Delta, France. J Vector ecology: J Soc Vector Ecol. 2012;37(2):269-75.

16. Liu FR, Han N, Zhang QW, Dong J, Liang XS. Analysis on mosquito monitoring and its influential factors in Longgang district of Shenzhen from 2008 to 2009. Practical Preventive Med. 2010;12:2393-6.

17. Yu XH, Lin D, Xu Y, Cao JH, Huang ZC, Zhu JG, Ni CR, Chen L, Xue DY. Light-trap surveillance of the community structure and seasonal fluctuation of mosquitoes in Wenzhou. Chinese J Vector Biol Control. 2010;01:68-9.

18. Fan W. A Study on the potential distribution of Aedes albopictus and risk forecasting for future epidemics of dengue in China. Master: Chinese Center For Disease Control And Prevention. 2009.

19. Liu CG, Luo L, Hu LF. Progress in the methods of monitoring mosquitoes. J Inspection Quarantine. 2013;1:52-7.

20. Lin LF, Duan JH, Li RB, Lin WB, Cai SW, Lu WC, Chen Q, Li JQ, Lin HB, Yi JR. Comparative study of the monitoring results of the Mosq-ovitrap method and the traditional larval index surveys in the field. Chinese J Vector Biol Control. 2006;06:454-7.

21. Sikulu M, Govella NJ, Ogoma SB, Mpangile J, Kambi SH, Kannady K, Chaki PC, Mukabana WR, Killeen GF. Comparative evaluation of the Ifakara tent trap-B, the standardized resting boxes and the human landing catch for sampling malaria vectors and other mosquitoes in urban Dar es Salaam. Tanzania Malaria J. 2009;8:197.

22. Gao Q, Zhou YB, Leng PE, Xiong CL, Jiang QW, Cao H, Huang P. Study on adult mosquito population dynamics by human landing catch in downtown Shanghai, China. Chinese J Vector Biol Control. 2014:03:215-8.

23. Maciel-de-Freitas R, Eiras AE, Lourenco-de-Oliveira R. Field evaluation of effectiveness of the BG-Sentinel, a new trap for capturing adult Aedes aegypti (Diptera: Culicidae). Mem Inst Oswaldo Cruz. 2006;101(3):321-5.

24. Schmaedick MA, Ball TS, Burkot TR, Gurr NE. Evaluation of three traps for sampling Aedes polynesiensis and other mosquito species in American Samoa. J Am Mosq Control Assoc. 2008;24(2):319-22

25. Meeraus WH, Armistead JS, Arias JR. Field comparison of novel and gold standard traps for collecting Aedes albopictus in Northern Virginia. J Am Mosq Control Assoc. 2008;24(2):244-8.

26. Bhalala H, Arias JR. The Zumba mosquito trap and BG-Sentinel trap: novel surveillance tools for host-seeking mosquitoes. J Am Mosq Control Assoc. 2009;25(2):134-9.

27. Wu JY, Lun ZR, James AA, Chen XG. Dengue fever in mainland China. AmJTrop Med Hyg. 2010;83(3):664-71.

28. Salazar FV, Achee NL, Grieco JP, Prabaripai A, Ojo TA, Eisen L, et al. Effect of Aedes aegypti exposure to spatial repellent chemicals on BG-Sentinel trap catches. Parasit Vectors. 2013:6:145.
29. Gouagna LC, Dehecq JS, Fontenille D, Dumont Y, Boyer S. Seasonal variation in size estimates of Aedes albopictus population based on standard mark-release-recapture experiments in an urban area on Reunion Island. Acta Trop. 2015;143:89-96.

30. Dong XS, Zhou HN, Gong ZD. The Mosquito Fauna Of Yunnan. 1st ed. Yunnan: Yunnan Science \& Technology Press; 2010.

31. de Azara TM, Degener CM, Roque RA, Ohly JJ, Geier M, Eiras AE. The impact of $\mathrm{CO} 2$ on collection of Aedes aegypti (Linnaeus) and Culex quinquefasciatus Say by BG-Sentinel(R) traps in Manaus, Brazil. Mem Inst Oswaldo Cruz. 2013;108(2):229-32.

32. Owino EA, Sang R, Sole CL, Pirk C, Mbogo C, Torto B. Field evaluation of natural human odours and the biogent-synthetic lure in trapping Aedes aegypti, vector of dengue and chikungunya viruses in Kenya. Parasites Vectors. 2014;7:451.

33. Farajollahi A, Kesavaraju B, Price DC, Williams GM, Healy SP, Gaugler R, Nelder MP. Field efficacy of BG-Sentinel and industry-standard traps for Aedes albopictus (Diptera: Culicidae) and West Nile virus surveillance. J Med Entomol. 2009;46(4):919-25.

34. Unlu I, Farajollahi A, Rochlin I, Crepeau TN, Strickman D, Gaugler R. Differences in male-female ratios of Aedes albopictus (Diptera: Culicidae) following ultra-low volume adulticide applications. Acta Trop. 2014;137:201-5.

35. Ree HI. Studies on Anopheles sinensis, the vector species of vivax malaria in Korea. Korean J Parasitol. 2005;43(3):75-92.

36. Diabate A, Tripet F. Targeting male mosquito mating behaviour for malaria control. Parasit Vectors. 2015;8:347.

37. Hartberg WK. Observations on the mating behaviour of Aedes aegypti in nature. Bull World Health Organ. 1971;45(6):847-50.

38. Howell PI, Knols BG. Male mating biology. Malar J. 2009;8 Suppl 2:S8.

39. Oliva CF, Damiens D, Benedict MQ. Male reproductive biology of Aedes mosquitoes. Acta Trop. 2014;132(Suppl):S12-19.

40. Zou HB, Song W, Lin HB, Li JL, Wang YF, Li ZQ, Liang HY, Zheng ZW, Yu Y Lin J. Background investigation on mosquitoes at Guangzhou ports along the Zhujiang river. Chinese J Frontier Health Quarantine. 2014;37(05):331-5.

41. Liu YS, Liang HY, Huang F, Li GH, Zhai JS, Zhang WD, Wu ZH, Chen J, Lin XH. Investigation on Mosquitoes at Luogang Ports in Guangzhou from 2008 to 2009. Chinese. J Frontier Health Quarantine. 2010;33:4.

\section{Submit your next manuscript to BioMed Central and we will help you at every step:}

- We accept pre-submission inquiries

- Our selector tool helps you to find the most relevant journal

- We provide round the clock customer support

- Convenient online submission

- Thorough peer review

- Inclusion in PubMed and all major indexing services

- Maximum visibility for your research

Submit your manuscript at www.biomedcentral.com/submit
Ciomed Central 